\title{
Robotic Pill for Biomarker and Fluid Sampling in the Gastrointestinal Tract
}

\author{
Fernando Soto ${ }^{1}$, Emma Purcell ${ }^{1}$, Mehmet Ozgun Ozen ${ }^{1}$, Prima Dewi Sinawag ${ }^{1}$, Jie Wang ${ }^{1}$, \\ Demir Akin ${ }^{1}$, and Utkan Demirci ${ }^{1}$ \\ ${ }^{1}$ Bio-Acoustic MEMS in Medicine (BAMM) Laboratory, Canary Center at Stanford for \\ Cancer Early DetectionDepartment of RadiologySchool of Medicine Stanford \\ UniversityPalo Alto, California
}

February 11, 2022

\begin{abstract}
Developing on-site biomarker enrichment platforms could help to improve the diagnosis of gastrointestinal (GI) tract diseases at early stages. Medical procedures, such as colonoscopies and imaging techniques, are used to diagnose disease, but are not easily accessible for repeat measurements. In the other hand, liquid biopsies, e.g., blood, urine, or fecal samples, have become important sampling strategies to identify health concerns. Herein, a robotic pill is designed for collecting relevant biomarkers from the GI over prolonged sampling periods. The robotic pill comprises a magnetic core for locomotion, a delayed gate mechanism that controls sampling location based on changes in its environment, and an enrichment module that traps biomarkers in an absorbent matrix while enabling biofluid to pass through the chamber. The robotic pill was assessed to sample microparticles, proteins, and bacteria from solution. Moreover, the robotic pill was capable of directed locomotion in complex environments and docking in a targeted region against fluid flow. Utilization of an untethered robotic sampling system could provide a tool to investigate aspects of disease initiation and progression for early diagnosis and therapy monitoring.
\end{abstract}

\section{ToC Figure}




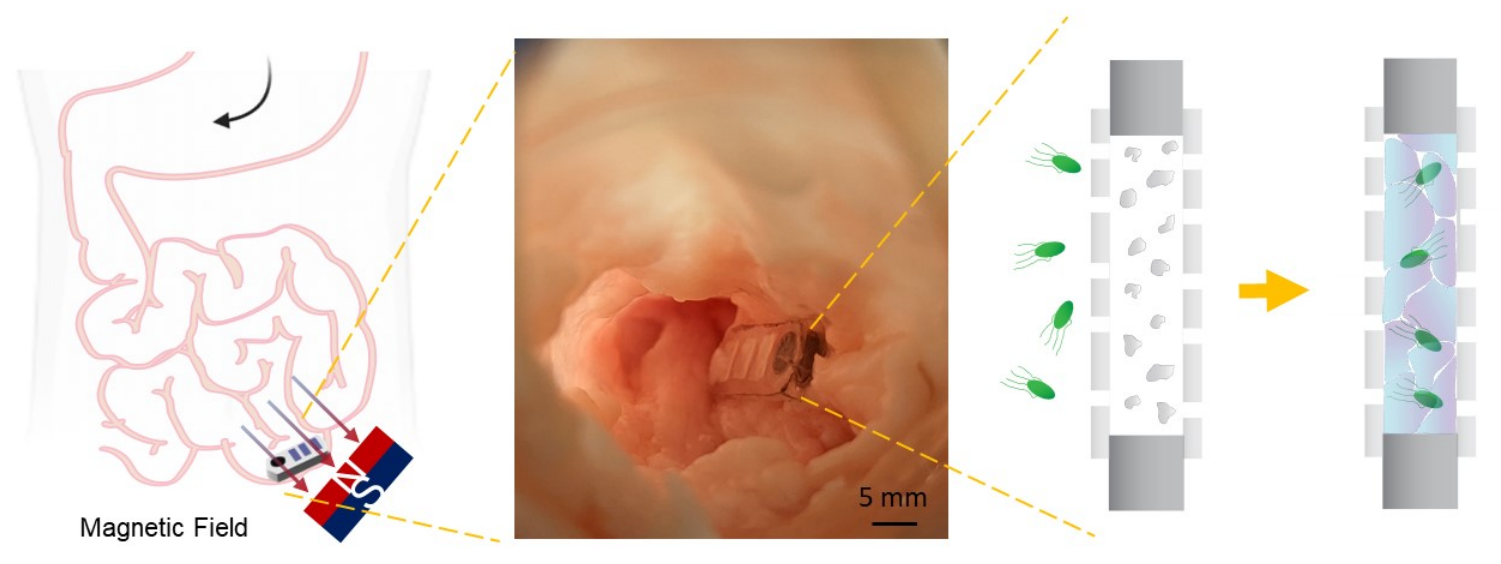

Figure 1: ToC Figure. Screening for cancer at early stages could improve disease prognosis and quality of life. Nevertheless, screening remains challenging for gastrointestinal cancers due to the limited accessibility. We report a robotic pill capable of delay opening of the collection chamber, sampling biomarkers (e.g. proteins and bacteria) from liquid samples and robust robotic control, including guidance through predetermined routes and docking against fluid flow.

\section{Introduction}

Health relevant information is coded in circulating signatures or biomarkers, such as the secretome, exosomes, nucleic acids, bacteria, and other markers for health.(Hay Burgess et al., 2006; Giljohann and Mirkin, 2009; Yuan et al., 2019) The collection and analysis of such signatures can serve as an early sign of disease initiation and its progression through repeated sampling.(Sinawang et al., 2021; Smith et al., 2002; Ransohoff, 2004; Ozen et al., 2020) Nevertheless, it remains a challenge to isolate or sample biomarkers from regions of the body with limited accessibility, such as the gastrointestinal tract, which is more than 30 feet in length in total.(Cummins et al., 2017; Badreddine and Wang, 2008) To gain access to these challenging locations, invasive medical procedures, such as surgery, biopsies, and colonoscopies, are required to extract information, limiting the repeatability of such measurements.(Washabau et al., 2010; Lhewa and Strate, 2012; Ciuti et al., 2020) These techniques involve trained practitioners, expensive instruments, and specialized facilities, making them hard to expand to a large population. On the other hand, minimally invasive approaches can also be used for sampling biomarkers.(Tang et al., 2020) For instance, liquid biopsy is a standard approach to obtain a snapshot of the signature pool from biofluids (blood, urine, or stool samples).(Saini et al., 2018; Wu et al., 2020) Although useful, liquid biopsy frequently provides indirect data that is averaged across the sample that is not specific to a particular region of the body. Smart pills have been deployed as a method to sample cavities inside the body.(Yim et al., 2014; Cummins, 2021; Mandsberg et al., 2020; Iddan et al., 2000; Min et al., 2019; Steiger et al., 2018) For instance, microfluidic chips,(Mimee et al., 2018; Qiao et al., 2016; Nemiroski et al., 2015) osmotic gradients,(Rezaei Nejad et al., 2019) and passive absorption(Waimin et al., 2020; Rios-Morales et al., 2021; Chen et al., 2020) have been deployed to collect biomarkers in the GI tract. Previous pill-based approaches are designed to focus on imaging of the GI tract(Iddan et al., 2000) or have active electrical components,(Schostek et al., 2016) and here we focus on capturing low abundance biomarkers via a simple. The ability to isolate biomarkers within localized environments and specificity could help discover novel early disease signals and monitor health status by allowing the generation of comprehensive datasets encompassing the shift from healthy to disease states and vice versa.

Herein, the use of a robotic pill platform is presented for enhanced collection and isolation of biomarkers. 
The robotic pill comprises three modular compartments with spatially resolved zones for different features, including sampling, delayed opening of the sampling gate, locomotion, and retention of the robotic pill in predetermined regions. The integration of these capabilities would assist in prolonged sampling at a target site, resulting in the capture of a sufficient quantity of GI biomarkers required to provide a sampling. The proposed approach could eventually result in a complementary sampling tool for routine gastric measurements in the clinical practice towards disease early detection and monitoring.

\section{Results and Disscussion}

The robotic pill comprised three modular compartments each designed for unique purposes as shown in Figure 2a, including the use of (i) s three sampling modules that allow the collection of different biomarkers in an absorbent trapping matrix while letting the biofluid pass through it, (ii) a porous membrane that enables the exclusion of larger objects $(>5 \mu \mathrm{m})$ in the collection chamber and (iii) a magnetic core embedded in the structure that aims to provide directed locomotion and retention of the robotic pill potentially at targeted regions of the body. The robotic pill prototype was fabricated using a laser cutter to cut optically clear and poly(methyl methacrylate) (PMMA) sheets (1.5-mm thick) into the pill shape with space for placing a 3 -mm neodymium magnet and three rectangular collection chambers $(3 \times 2 \mathrm{~mm}, 9 \mu \mathrm{L}$ per chamber $)$. The plastic backbone provides mechanical stability to the pill structure and holds the absorbent material in the collection chamber. A double-sided adhesive film was cut in the shape of the robotic pill backbone and used to adhere a polycarbonate membrane with $5 \mu \mathrm{m}$ pores to the plastic backbone. Sodium polyacrylate powder (absorbent) was placed in the collection chamber before sealing the collection chambers on both sides using the polymeric membrane. (Figure S1). Integrating absorption, delayed opening of the collection chamber, and docking control into a single device could potentially enable the isolation of biomarkers within the hardto-access regions of the body, as shown in schematic illustrating the retention of a robotic pill in the small intestine through magnetic retention (Figure $\mathbf{2 b}$ ). In addition, the pill was designed to be comparable to FDA-approved ingestible device standards, as its measurements are $16 \mathrm{~mm}$ in length, $6 \mathrm{~mm}$ in diameter and $1.5 \mathrm{~mm}$ in thickness.(Sayeed, 2015) (Figure 2c). The sampling capability of the pill is illustrated by Figure 2d, where the photographs show a robotic pill before (top) and after (bottom) immersion (5 min) in water colored in blue dye, where each of the three collection chambers retained the liquid captured by the absorbent material.

The robotic pill collection chambers are designed to entrap biomarkers within an absorbent polymeric matrix while enabling fluid to permeate through it. The sodium polyacrylate enclosed between the porous membranes absorbs an aqueous solution that permeates through the membrane. The hydrogen bond interactions between the carboxylic groups of the absorbent material and the surrounding water molecules results in the expansion of the polymeric matrix and the untangling of the polymeric chains, creating swelling as liquid is absorbed (Figure 3a).(Liu et al., 2019) The absorbent matrix dynamically increases biomarker retention in the device, while fluid permeates through the collection chamber. The resulting expansion and swelling enable the physical entrapment of biomarkers in between the swollen hydrogel's matrix. Instead, without the use of the hydrogel matrix, both the liquid and biomarkers would leak freely. The dry absorbent powder can expand rapidly $\left({ }^{\sim} 10 \mathrm{sec}\right)$ to form a large hydrogel matrix, as shown in Figure S2 and Video S1.

Rich media available at https://youtu.be/hbkfucVlW_U

The swelling behavior can be attributed to competing interactions between the ions present in the gastric simulant and water molecules with the polymeric backbone of the absorbent material. The material's 

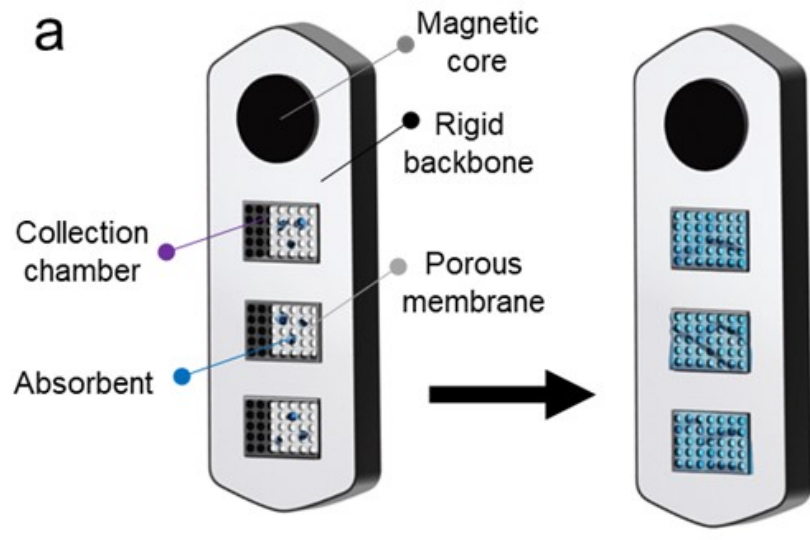

b

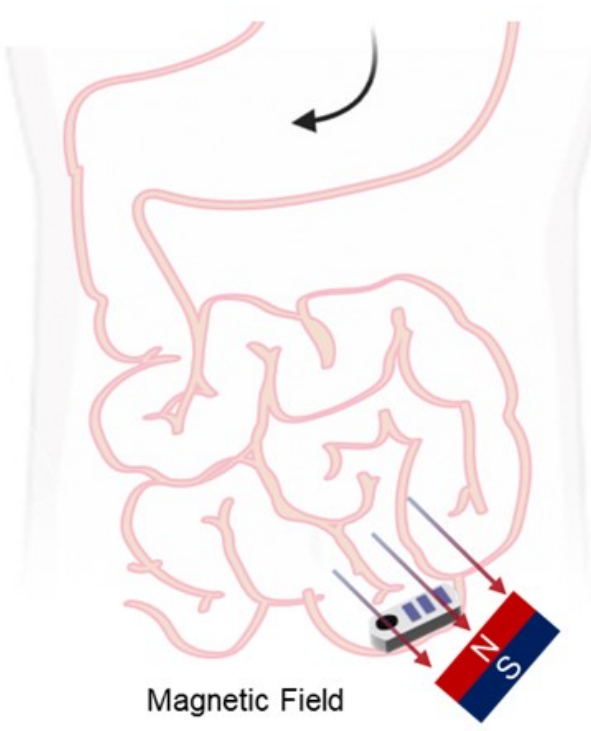

i) Absorbent

ii) Porous membrane

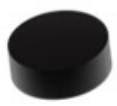

iii)Magnet
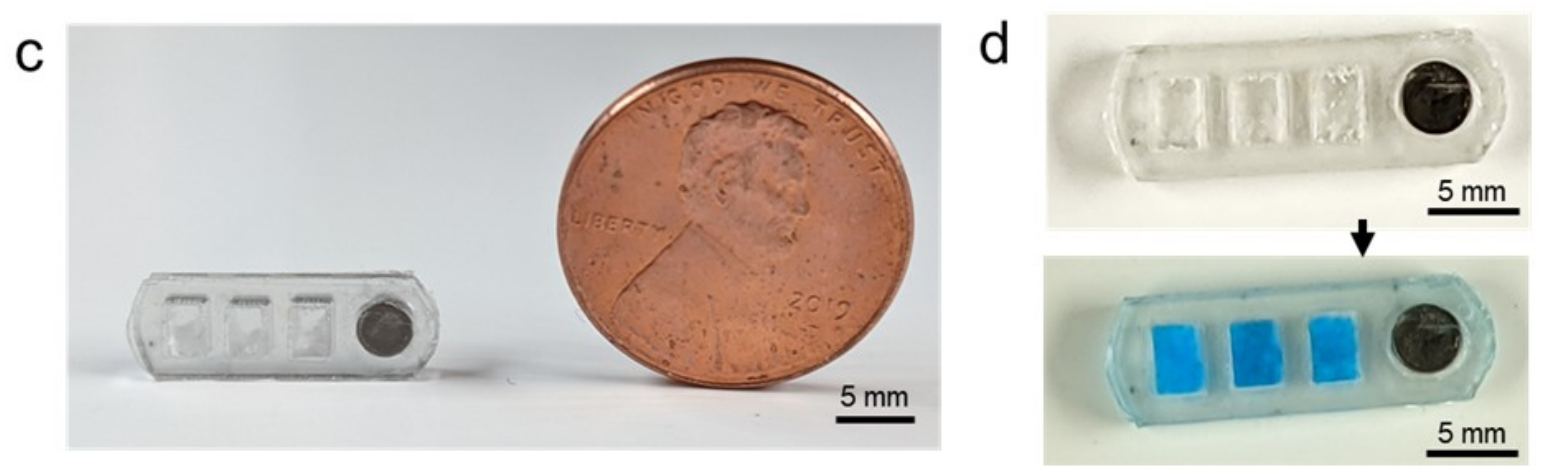

Figure 2: Robotic pill for sampling body cavities. a) Scheme of robotic pill illustrating main components including i) an absorbent material to capture biomarkers and biofluids, ii) a pH-sensitive porous membrane and iii) a magnetic core that enables navigation and retention. b) Scheme illustrating docking of the robotic pill within the intestines in the presence of an external magnetic field. c) Photograph illustrating the actual size of the robotic pill. (16 6 × $1.5 \mathrm{~mm}$ ) d) Photograph illustrating sampling liquid solution using a robotic pill, the top image illustrates dry robotic pill and bottom the same robotic pill after 1-minute incubation in a blue dye solution.

absorption property was evaluated by measuring the mass swelling capacity, that translates to the amount of fluid contained per gram of absorbent material (Figure S3). For instance, the absorbent material captured $45.97 \pm 1.71 \mathrm{~g}$ of DI water/g absorbent, $44.62 \mathrm{~g} / \mathrm{g} \pm 0.85$ of buffer solution and, $34.90 \mathrm{~g} / \mathrm{g} \pm 1.99$ of gastric simulant. The absorbent material is hydrophilic, hence it quickly interacts with the water molecules in aqueous solutions. This interaction results in the expansion of the coiled network of the polymer, and consequent swelling (Figure 3a). The polymer-solvent interactions can then be tuned based on the solution properties, such as ionic strength or $\mathrm{pH}$, that will determine the solvent quality, water/polymer interactions, and consequent swelling/de-swelling behavior. This phenomenon can be employed to provide reversible deswelling of the absorbent matrix by placing the fully swollen hydrogel matrix in a high concentration of 
a
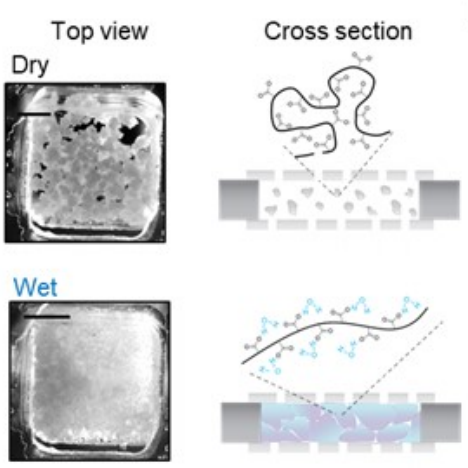

d

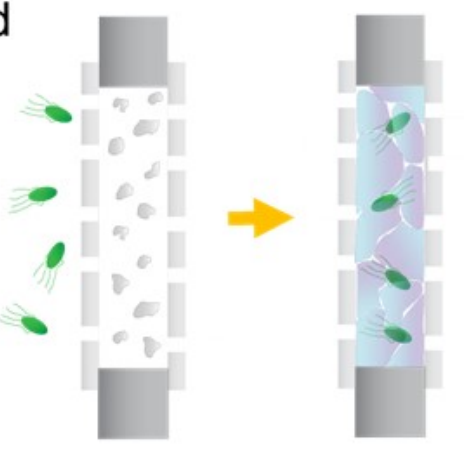

b
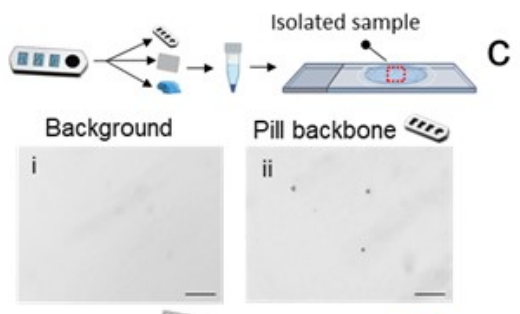

Membrane

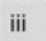

iii

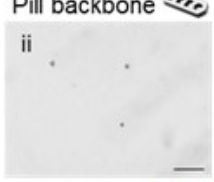

Absorbent

iv

3

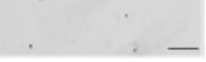

e

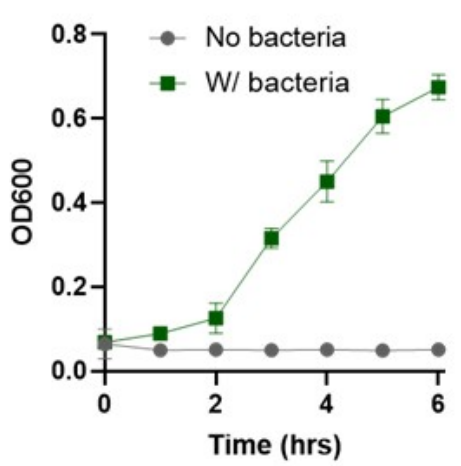

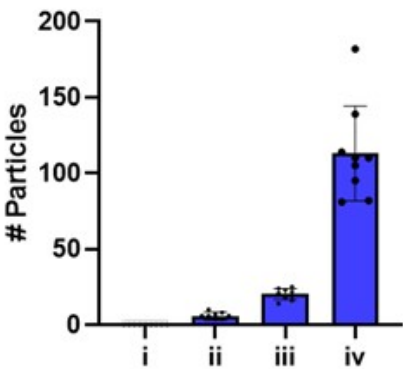

f

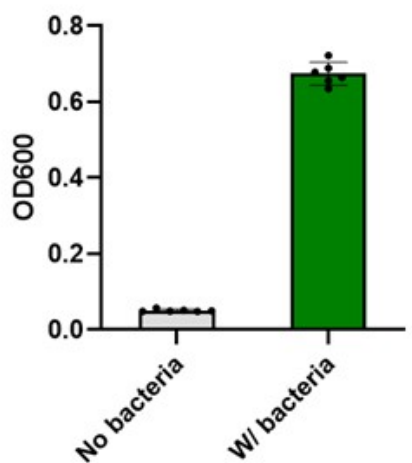

Figure 3: Absorbent material-based capture and isolation. a) Scheme and images of the swelling-based enrichment module before and after capture. Scale bar: $0.5 \mathrm{~mm}$. b) Micrographs illustrating $2 \mu \mathrm{m}$ microparticles recovered from the collector from different parts of the pill, (i) background (control pill with no beads), (ii) on the plastic backbone, (iii) within the porous membrane, and (iv) within the absorbent material. Scale bar: $80 \mu \mathrm{m}$ c) Quantification of particles retained at the stages described in panel $b(n=9)$. d) Scheme of the robotic pill capturing bacteria. e) Optical density measured at a wavelength of $600 \mathrm{~nm}$ (OD600), quantifying the growth of bacteria recovered by robotic pill after a 1-hour incubation ( $n=6)$. f) Quantification of E. coli growth after 6 hours $(\mathrm{n}=6)$.

specific ions (e.g., Na+ or $\mathrm{Ca} 2+$ ), as illustrated in Figure S4. The osmotic pressure caused by ions in solution leads to a shift in water molecules toward the more hypertonic environment, leaving the polymeric chains free to coil back to their original position, resulting in hydrogel de-swelling, and heightened biomarker release.(Liu et al., 2019)

To demonstrate that liquid-based biomarkers are specifically contained within the absorption matrix, the specific contribution of each component of the robotic pill to biomarker uptake was evaluated. Fully-assembled robotic pills were incubated for one hour in a solution containing $2 \mu \mathrm{m}$ beads under constant shaking. Following the incubation, the pills were disassembled into their components, and the capture contribution of each component was analyzed by measuring the number of beads recovered from each separate robotic pill component, including (i) a negative control pill incubated without beads, (ii) the plastic backbone, (iii) porous membrane, and (iv) absorbent material, as shown in Figure 3b. These measurements indicate that the absorbent material provides a larger number of beads trapped $(113 \pm 29)$ when compared to the plastic backbone $(6 \pm 3)$, and porous membranes $(20 \pm 4)$ (Figure 3c). The sum of all beads recovered by the different pill components represents a capture yield of 29.8 percent from stock solution. In addition, the 
release of the microbeads was assisted using a $2 \mathrm{M} \mathrm{CaCl} 2$ solution that helped to deswell the gel and release the trapped particles. We should note that this release method would help accelerate processing time but could affect biological targets viability after release. Figure S5 illustrates the colorimetric quantification of the recovered bovine serum albumin (BSA) protein captured overnight by a pill collector under different recovery protocols, including i) placing the absorbent material in a $2 \mathrm{M} \mathrm{CaCl} 2$ solution and shaking for 30 min, and i) placing the absorbent material in a PBS solution and shaking for $30 \mathrm{~min}$. Although both batches were incubated following the same protocol, the presence of a salt-rich environment promoted precipitation of the recovered protein affecting the analytical measurement compared to the use of buffer solution. Thus, the recovery method can be tailored for a different type of biomarkers.

The robotic pill was also evaluated for the capture of motile bacteria. The expansion of the absorbent material can serve to trap the bacteria within the three-dimensional porous polymer matrix,(Di Giacomo et al., 2017; Bhattacharjee and Datta, 2019) retaining them in the structure under a moist environment, as shown in the scheme in Figure 3d and Video S2.

Rich media available at https://youtu.be/XyDU-s8ca_g

The robotic pill was incubated for one hour in a solution containing E. coli at a starting OD600 of $1.48 \pm 0.04$ $(\mathrm{n}=3)$, indicating high turbidity and bacteria quantity. After incubation, the absorbent material inside the robotic pill was transferred to a solution containing growth media and was measured every hour to evaluate its growth (Figure 3e). A control study was performed incubating a robotic pill in growth media without bacteria to test for contamination. After 6 hours, the control robotic pill presented a background OD600 of $0.05 \pm 0.003$ when compared to $0.67 \pm 0.03$ for the pill incubated with bacteria, illustrating the ability for the pill collector to trap and retain living bacteria (Figure 3f).

The porous membrane of the robotic pill was coated with polymeric enteric-alike coating, (poly(meth)acrylate), which dissolves under physiological environmental $\mathrm{pH}$ conditions found in certain locations in the GI tract, thus enabling the enrichment module to open in the GI tract rather than in the stomach.(Xiong et al., 2020; Li et al., 2016; Wu et al., 2019; Soto et al., 2021) A table of pH values at various locations at the stomach and GI tract is presented in Table 1.(Evans et al., 1988)

$\begin{array}{cc}\text { Gastrointestinal Region } & \mathrm{pH} \\ \text { Stomach } & 1.0-2.5 \\ \text { Proximal small intestine } & 6.6 \\ \text { Terminal ileum } & 7.5 \\ \text { Caecum } & 6.4 \\ \text { Colon } & 7\end{array}$

Table 1: Mean pH levels in the gastrointestinal tract sections from normal subjects.(Evans et al., 1988)

The coated porous membrane was used to mimic the enteric coating of the GI tract. In this experiment, it contained fluorescent dye to more easily evaluate the selective opening of the coated pores in gastric and intestinal model fluids. Figure 4a shows images of the porous membrane in the following conditions, i) without coating, ii) filled with an enteric coating containing a fluorescent dye, iii) filled with fluorescent enteric coating after one-hour incubation in gastric simulant ( $\mathrm{pH} \mathrm{2.0)}$ ) and, iv) filled with fluorescent enteric coating after one-hour incubation in buffer $(\mathrm{pH}$ 7.4). The coated membrane immersed in gastric simulant (low $\mathrm{pH}$ ) maintained the polymeric coating blocking the pores, while the enteric-coated membrane submerged in buffer solution (neutral $\mathrm{pH}$ ) dissolved, Figure $\mathbf{4 b}$. The ability to delay the sampling of the robotic pill was evaluated by incubating the coated pill in a fluorescent solution. In this case, the enteric coating was not loaded with the fluorescent dye; thus, the signal obtained was related to the captured solution. Figure 4c 
a
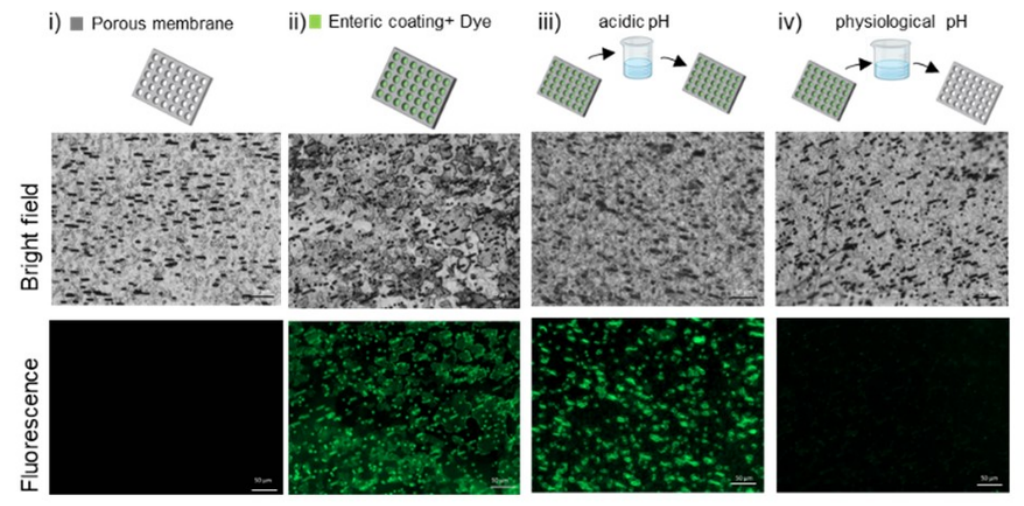

b
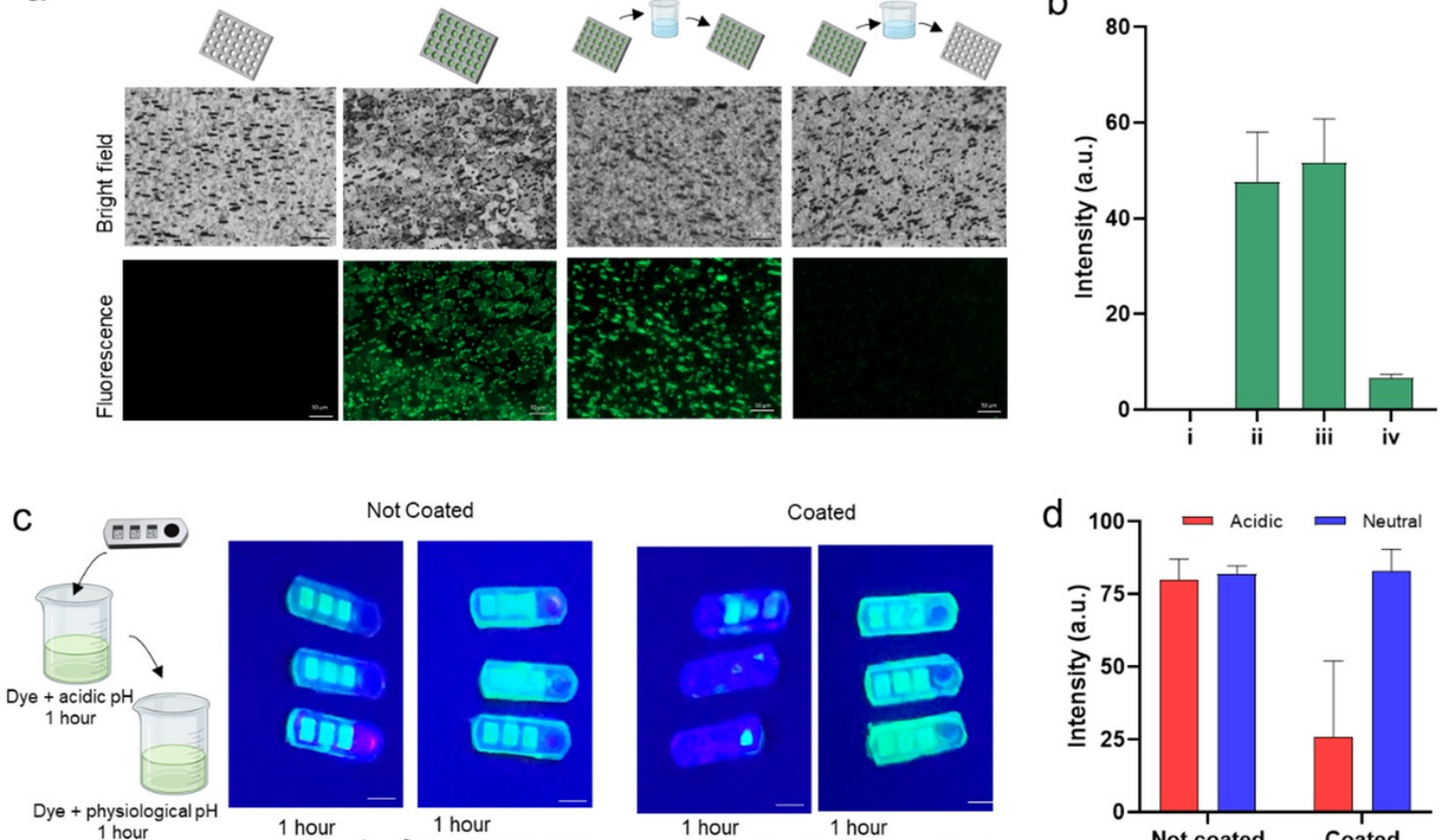

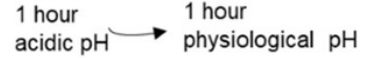

Not Coated

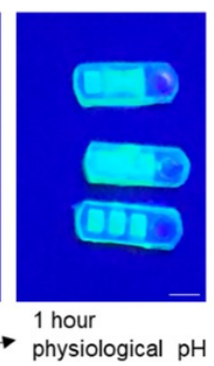

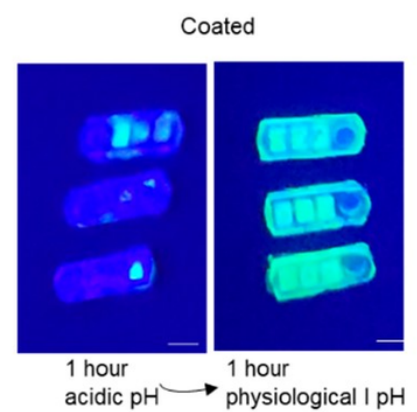

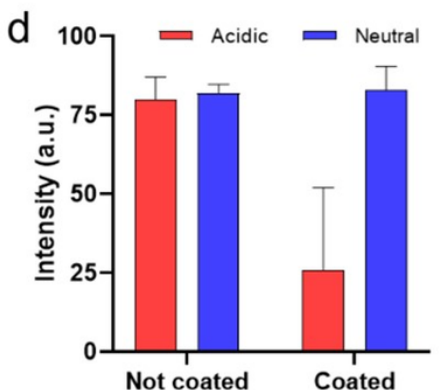

Figure 4: pH-sensitive opening of collection chamber in the robotic pill. a) Scheme, bright field, and fluorescent images of membrane pores of the robotic pill, i) without coating, ii) filled with an $\mathrm{pH}$ sensitive enteric coating containing a fluorescent dye, iii) filled with fluorescent enteric coating after one-hour incubation in gastric simulant ( $\mathrm{pH} \mathrm{2}$ ) and, iv) filled with fluorescent enteric coating after one-hour incubation in buffer (pH 7.4). Scale bar: $75 \mu \mathrm{m}$. The enteric coating contains fluorescent green dye for contrast. Scale bar: 50 $\mu \mathrm{m}$. b) Quantification of fluorescent intensity in $\mathrm{b}(\mathrm{n}=10)$. c) Photographs under fluorescent light showing the comparison of absorbent-loaded robotic pills i) with coating and ii) without coating after sequential incubation in acidic $\mathrm{pH}$ and neutral $\mathrm{pH}$ solutions containing fluorescent dye solution. Scale bar: $5 \mathrm{~mm}$ e) Quantification of fluorescent intensity in $\mathrm{d}(\mathrm{n}=9)$.

displays photographs under fluorescent light of i) a robotic pill with no coating and ii) a coated robotic pill after one-hour incubation in gastric simulant with fluorescent dye and after the subsequent incubation of the same pill in buffer media. The non-coated porous membrane pill absorbed the media during incubation in the acidic solution. Conversely, the coated porous membrane presented limited absorption after incubation in acidic medium and full absorption at physiological pH, as shown in Figure 4d. These results indicate the potential of the robotic pill to selectively open its collection chamber in the intestine rather than in the stomach, thus avoiding contamination with stomach biomarkers.

The robotic pill was capable of directed locomotion in complex environments. When placed in the presence of an external magnetic field, the magnetic core embedded in the robotic pill can apply torque force to the whole pill structure to reorient according to the magnetic field lines from the external actuator (Figure 5a). This behavior can actuate the robotic pill in a walking propulsion mechanism, as shown in Figure 5b. The robotic pill is rotated along its longitudinal axis, producing displacement by each magnetic field turn.(Tu and Gao, 2020; Hu et al., 2018; Lee et al., 2022; Xu et al., 2022; Sayed et al., 2022; Kosa and Hunziker, 2019) The robotic pill can be guided through predetermined paths, as illustrated in Figure 5c, which shows the 
overlap of multiple images taken from Video S3.

Rich media available at https://youtu.be/H4MXTZ6F3nk

This locomotion mechanism can be used to navigate the structure over complex and uneven routes. For instance, Figure 5d and Video S4 rocks show the overlap of a pill's trajectory moving in an uneven terrain composed of randomly dispersed small rocks. If the robotic pill was dragged by a magnetic attraction, it would get stuck in the uneven surface of the terrain; thus, the walking propulsion mode enables it to avoid lodging in between the rocks, roughly imitating the crevices found within the GI tract.

Rich media available at https://youtu.be/wAzv8wAG188

The robotic pill locomotion was also tested over an obstacle course containing a ramp and two elevated platforms separated by a small gap. The robotic pill was guided to go up the ramp at different elevation. (Figure 5e and Video S5).

Rich media available at https://youtu.be/01cdeCn6F00

The actuation response of the robotic pill over biological samples was demonstrated by making it walk over a $20 \mathrm{~mm}$ thick belly strip, indicating the ability to actuate the robotic pill across tissues (Figure $\mathbf{5 f}$ and Video S6).

Rich media available at https://youtu.be/rXAUWM4eS-Y

The robotic pill design can also perform more complex tasks apart from simple locomotion. This is illustrated in Figure 5g and Video S7 where the robotic pill showed transport and reorganization of three cubes by pushing them in predetermined locations.

Rich media available at https://youtu.be/j7e7Ck3ZNq0

Moreover, multiple robotic pills can be actuated at the same time and be assembled into larger structures, Figure $\mathbf{5 h}$ and Video S8. Here four identical but separate robotic pills were assembled into a single larger structure, indicating the possibility of creating larger modular structures by assembling building blocks of individual robotic pills with different functions such as coatings, pore sizes or targets.

Rich media available at https://youtu.be/KdRE5aDJRMs

The ability of the pill to dock for increased time in a target region of the GI would improve the sampling of locally generated biomarkers.(Iacovacci et al., 2021; Liu et al., 2021; Soto et al., 2020; Laulicht et al., 2011) The retention and docking abilities of the robotic pill were evaluated under constant fluid flow in both a small intestine tubing model and a porcine small intestine.

Figure 6a illustrates a scheme and photographs of a robotic pill, passing through a plastic GI model under a flow of 7.2 liters per min, i) without a magnetic field, and ii) with a magnetic field. Figures $\mathbf{6 b}$ and $\mathbf{6 c}$ illustrate the displacement and speed of the robotic pill under each condition (magnetic field vs no magnetic field), demonstrating that the robotic pill can be docked in a specific location and retain at the target location through magnetic force (Video S9). 
Rich media available at https://youtu.be/tHJ71VV3XfI

Moreover, the pill can be reoriented in different positions even in active flow (Figure $5 \mathrm{~d}$ ) and move against flow to reposition to a desired location against flow (Figure S6 and Video S10).

Rich media available at https://youtu.be/RJhPfme45m8

Finally, the retention capabilities of the robotic pill were tested using a real porcine intestine model, Figure 6e. A schematic of the robotic pill being docked in a section of the intestine by interaction with a magnetic field is demonstrated by Figure 6f. The robotic pill was small enough to travel through the intestine and be retained at specific locations, as illustrated by Figure $\mathbf{6 g}$. The dynamic retention of the robotic pill using the porcine intestine model was also evaluated under constant fluid flow, where the intestine was connected to a mechanical pump and subjected to continuous fluid flow (Figure S7). The pill docked at the region next to the magnetic field for minimum half an hour of constant flow (7.2 liter per min). The pills were also capable of changing position under magnetic guidance, as shown in Figure 6h. We note that the robotic pill can deform the tissue while being dragged toward the permanent magnet, but it did not generate any tearing or leakage. The ability of the pill to dock at a specific location potentially provides a platform for long-term sampling inside the body. 

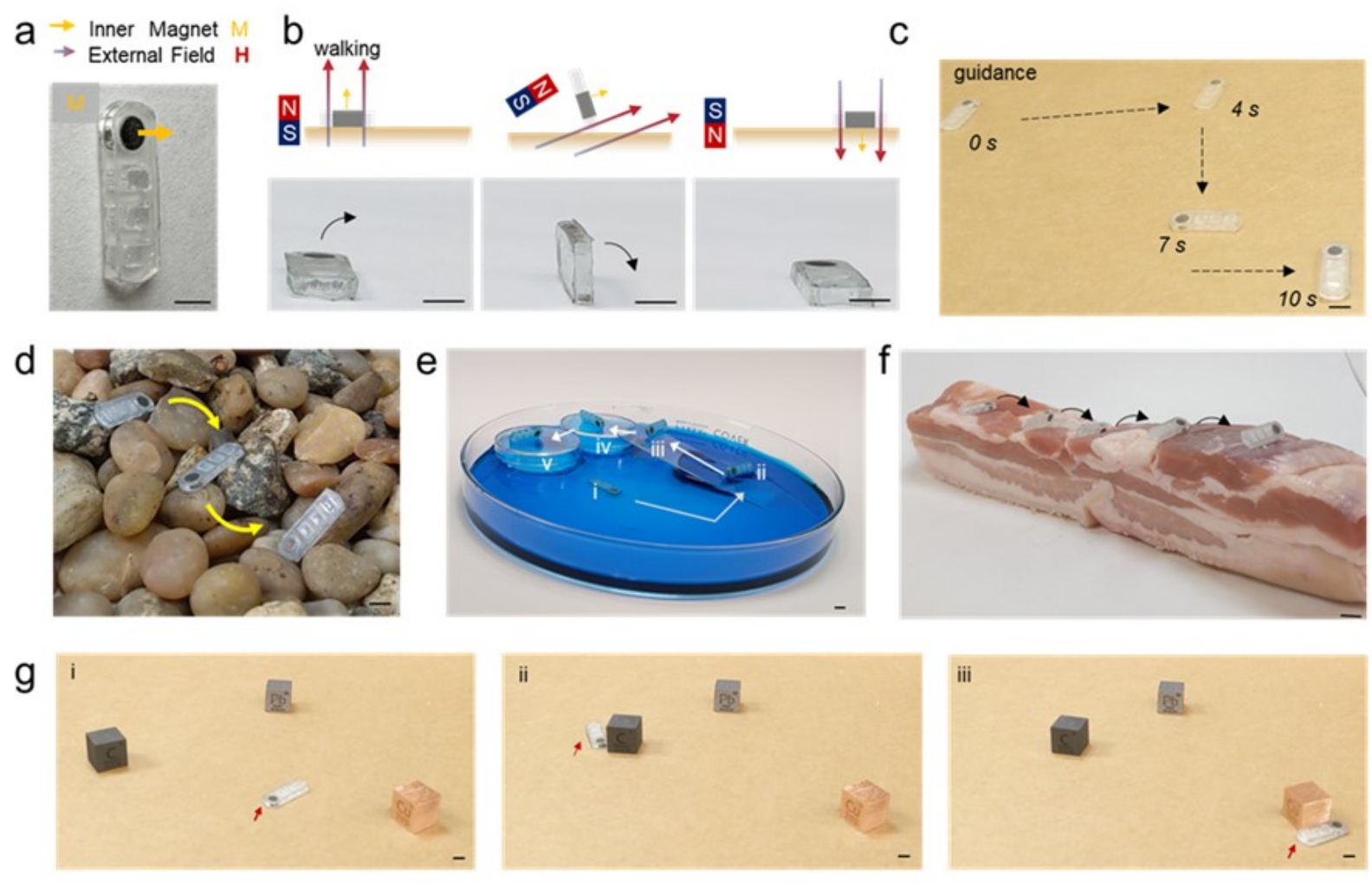

d
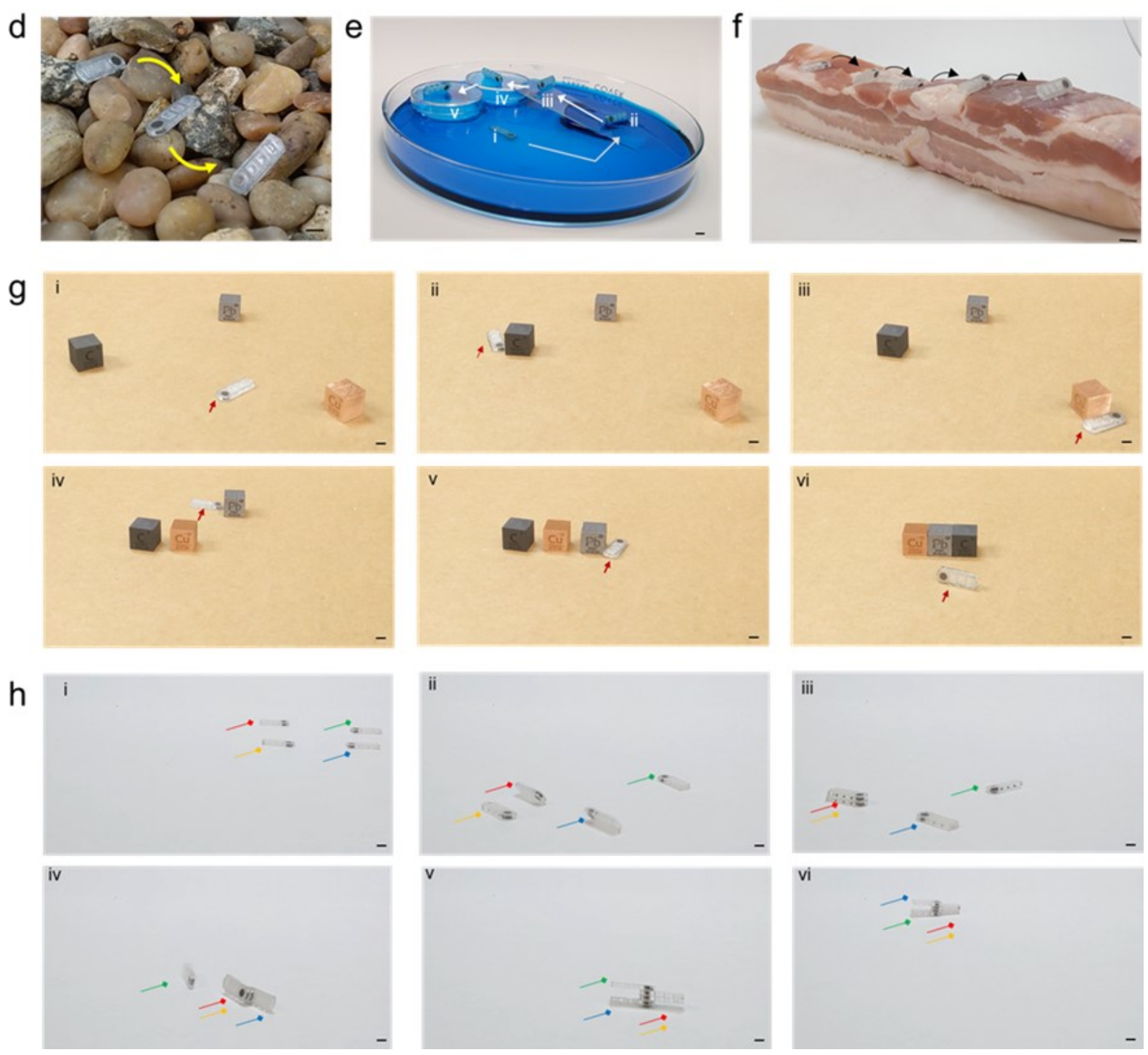

Figure 5: Locomotion of robotic pill. a) Photograph of the pill illustrating the inner magnetic field direction (M). b) Scheme and photograph of the walking locomotion mechanism based on the response to an external magnetic field (H). c) Time-lapse image of directed locomotion of a pill. d) Locomotion of a robotic pill through rough terrain composed of small rocks. e) Robotic pill locomotion through an obstacle course containing ramps and two elevated platforms separated by a gap. f) Locomotion of a robotic pill over a pork belly strip. g) Time lapse images (i-vi) of robotic pill to transport and rearrange small cubes. h) Simultaneous actuation of four robotic pills and their assembly into a larger structure. Scale bar: $5 \mathrm{~mm}$. 

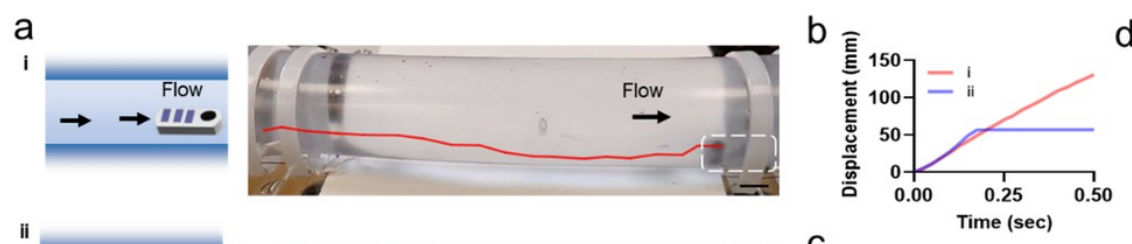

ii
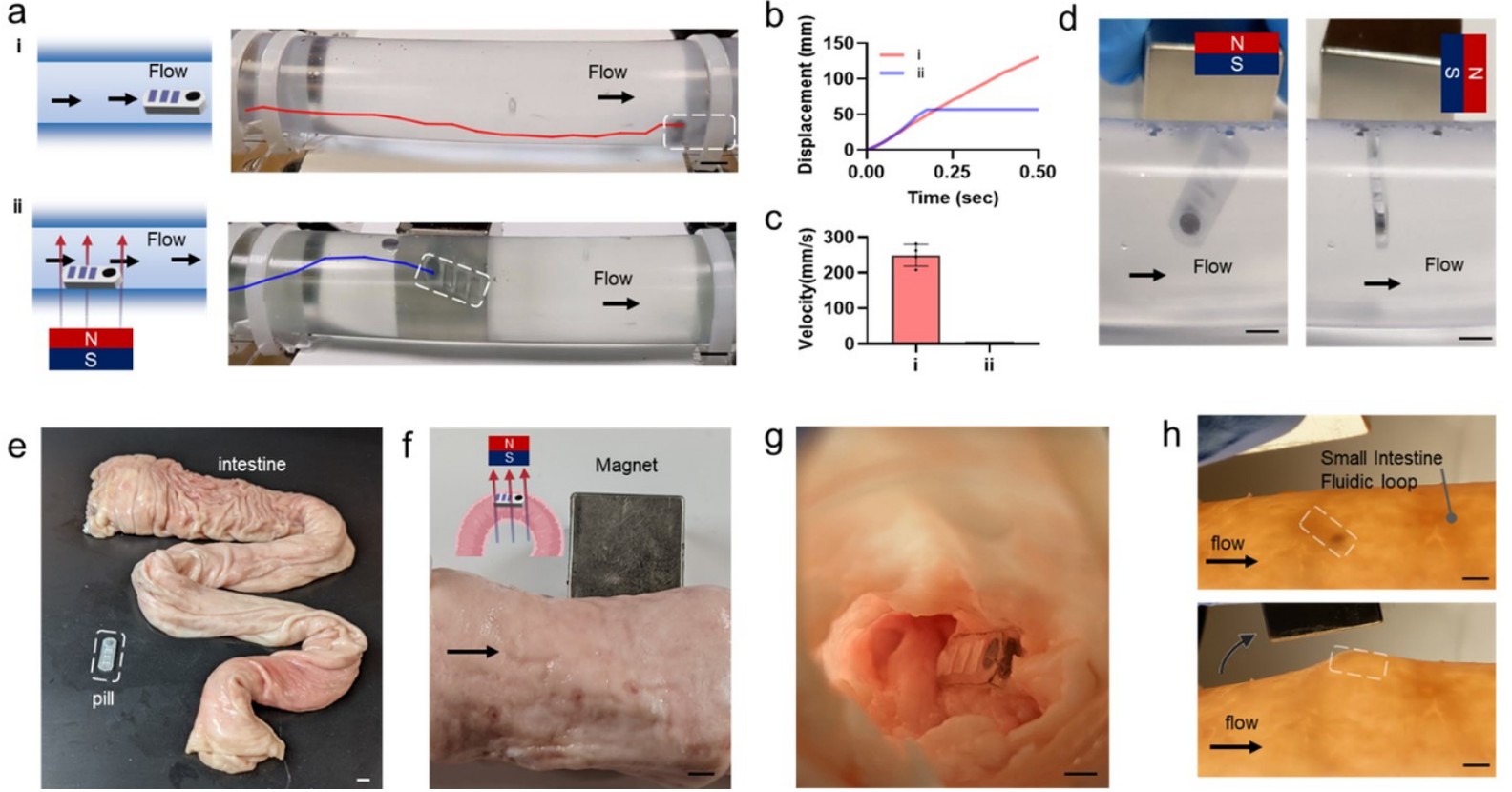

Figure 6: Figure 5. Docking of robotic pill at a target location under flow conditions. a) Scheme and photographs of pill being held in one position in a small intestine tube model under flow conditions (i) without and (ii) with an external magnetic field. b) Displacement and c) speed quantification of robotic pill, under i) no magnetic field, ii) magnetic field under constant fluid flow in the small intestine tubing model. d) Photographs illustrating different orientations of a pill by rotating the external magnetic field. e) Photograph illustrating robotic pill next to a porcine small intestine for scale. f) Photograph scheme illustrating the use of an external magnetic field to localize the robotic pill in a specific small intestine location. g) Photograph of a robotic pill inside the intestine. h) Photograph of robotic pill being transported and docked in a specific location within a small intestine during active fluid flow. Scale bars: $5 \mathrm{~mm}$. 


\section{Conclusion}

In this work, a robotic pill was evaluated as a proof-of-concept biomarker sampling platform. The robotic pill was fabricated by incorporating simple materials commonly used in medical applications (e.g., PMMA, porous membranes, sodium polyacrylate). Experiments were performed to evaluate the robotic pill's ability to entrap microparticles, proteins, and bacteria as model target collectable biomarkers. Moreover, the delayed opening of the collection chamber was achieved by coating the porous membrane with an enteric like coating which was shown to delay collection in acidic environments while opening in subsequent incubation in physiological $\mathrm{pH}$ environments. The robust robotic locomotion, guidance in complex environments and docking for a prolonged time in a porcine intestine model under constant flow was also demonstrated.

There are still aspects that can be enhanced within the current platform. For example, the continuous sampling provided by this design increases the possibility of cross contamination with other regions of the GI compared to other methods that collect fluid at one timepoint and keep their collection chamber sealed off. This device can be fabricated inexpensively when compared to other more robust robotic pills which integrate electronic or moving components. Therefore, our proposed model could be used to explore and sample low-abundance biomarkers in resource-limited environments, owing to its simple design and relatively inexpensive cost. For instance, the pill contents could be evaluated with downstream omics and colorimetric assays, moving this technology towards improved biomarker sampling.

In the next phase, the robotic pill control system will be integrated with automated guidance and imaging providing a higher degree of control and data collection. Moreover, we plan to study other relevant biomarkers, such as extracellular vesicles and the gut biome. We expect that the robotic pill collector would not present any risk to the user, as its small size and shape have been widely used in other ingestible imaging pills.(Steiger et al., 2018) The principles outlined by this work could provide the foundation for a rapidly adaptable platform capable of isolating signatures for disease detection at early stages and therapy monitoring at later stages.

\section{Experimental Section/Methods}

Robotic Pill Fabrication: The robotic pill was fabricated by integrating several components, including: (1) a $1.5 \mathrm{~mm}$ laser cut optically clear cast acrylic sheet (Mcaster-carr) as a rigid backbone, (2) a 5 um cylcopore polycarbonate membrane (Whatman) manually cut from $1.5 \mathrm{~mm}$ plastic sheet adhered to the rigid backbone using a double-sided adhesive medical (ARcare@90445, Adhesives Research) tape with the shape of the rigid backbone, (3) a cylindrical neodymium magnet $3 \mathrm{~mm}$ in diameter and $1.5 \mathrm{~mm}$ in thickness (K\&J Magnetics, 6451 Gauss) and (4) sodium polyacrylate (Caroline biological supply) as the absorbent materials.

Quantifying the microparticle, protein, and bacteria uptake of the pill: The material's absorption was characterized by adding $500 \mu \mathrm{L}$ of water, phosphate buffer saline (PBS), and gastric simulant to $10 \mathrm{mg}$ of dry absorbent powder. The powder absorbed the solution and was weighed to measure the captured solution and compared to the original weight of the added solution $(500 \mu \mathrm{g})$ for normalization. To evaluate the capture contribution of each of the pill's components, the robotic pill was incubated in a $1 \mathrm{~mL}$ solution containing $2 \mu \mathrm{m}$ microbeads (Spherotech, $465 \pm 57$ particles $/ \mu \mathrm{L}$ ). After incubation, the different parts of the robotic pill (rigid backbone, membrane, absorbent material) were placed in a $1 \mathrm{~mL}$ solution and gently rotated for $15 \mathrm{~min}$. $1 \mu \mathrm{L}$ of the supernatant was pipetted onto a glass slide, The collection capability was analyzed by measuring the number of particles in the $200 \times 200 \mu \mathrm{m} 2$ area located at the center of each droplet using brightfield microscopy, and quantified using the ImageJ count function. 
For protein analysis, the robotic pill was incubated overnight in 1\% BSA solution in PBS. After collection, the absorbent material was removed from the robotic pill structure and placed inside a container with either PBS or $2 \mathrm{M} \mathrm{CaCl2}$. The amount of protein released from the absorbent material was quantified using microBCA (Pierce) using the microplate standard protocol with the samples at a 1:10 dilution. Similarly, to quantify bacterial uptake, the robotic pill was incubated with E. coli (ATTC, 25922GFP) at a starting concentration of 1.5 OD600 for an hour under shaking conditions. The three absorbent gel matrixes were removed from the pill collector, and each placed in separate $2 \mathrm{ml}$ of growth media for evaluating growth. $200 \mu \mathrm{L}$ was removed from each tube every hour for 8 hours to quantify bacterial growth as measured using OD600.

Evaluating the effects of coating the pill membrane: The porous membrane was coated with a EUDRAGITß L 100-55 (Evonik) using manufacturers protocols and drop cast over a porous membrane containing a plastic adhesive in the shape of the robotic pill. The borders of the adhesive layer help to contain the enteric solution in place. The material was left to dry overnight before use. UV green dye (Bitspower) was used to provide contrast in the evaluation of the presence of enteric coating in the porous membrane after incubation and as an indicator captured solution. The membranes and pills were placed in either simulated gastric fluid simulant (Ricca) or buffer solution.

Demonstrating pill locomotion using an external magnetic field: A 1.5-inch square neodymium magnet (K\&J Magnetics) was placed $20 \mathrm{~mm}$ below a table substrate supporting the robotic pill to induce locomotion. The mangetic filed strength was measured at different distances from the surface of the magnet by a handheld digital teslameter (TD8620,Tunkia) as shown in Figure S8. The magnet was rotated manually to cause the rotation of the robotic pill. The robotic pill was forced to walk across small stones $(<10 \mathrm{~mm})$ and an inch thick porcine belly strip (bought at the local supermarket). For fluidic experiments, either a one-inch diameter plastic tube or porcine intestine (bought at the local market) were connected to a fountain pump to generate a closed-loop fluidic system. The data that support the findings of this study are available from the corresponding author upon reasonable request.

\section{Acknowledgements}

This work was supported by the Stanford RISE COVID-19 Crisis Response Seed Grant Program, and the Canary Center at Stanford for Cancer Early Detection Seed Award. F.S was supported by the Stanford Molecular Imaging Scholars program, 5R25CA118681. (NIH T32 postdoctoral fellowship) and the Schmidt Science Fellows in partnership with the Rhodes Trust. P.D.S. was supported by the James D. Plummer Graduate Fellowship, EDGE Doctoral Fellowship Program, the Dean's Office of the Stanford School of Engineering, and the Department of Chemical Engineering. The authors thank C. F. Guimarães for the helpful discussion.

\section{Conflict of interest}

Prof. Utkan Demirci (UD) is a founder of and has an equity interest in: (i) DxNow Inc., a company that is developing microfluidic IVF tools and imaging technologies, (ii) Koek Biotech, a company that is developing microfluidic technologies for clinical solutions, (iii) Levitas Inc., a company focusing on developing microfluidic sorters using magnetic levitation, (iv) Hillel Inc., a company bringing microfluidic cell phone tools to home settings, and (v) Mercury Biosciences, a company developing vesicle isolation technologies. UD's interests were viewed and managed in accordance with the conflict-of-interest policies. 


\section{References}

Rami Badreddine and Kenneth K. Wang. Biomarkers in Gastrointestinal Cancers. The American journal of gastroenterology, 103(8):2106, aug 2008. ISSN 00029270. doi: 10.1111/J.1572-0241.2008.01994.X. URL /pmc/articles/PMC2664740/https://www.ncbi.nlm.nih.gov/pmc/articles/PMC2664740/.

Tapomoy Bhattacharjee and Sujit S. Datta. Bacterial hopping and trapping in porous media. Nature Communications 2019 10:1, 10(1):1-9, may 2019. ISSN 2041-1723. doi: 10.1038/s41467-019-10115-1. URL https://www.nature.com/articles/s41467-019-10115-1.

Lu Chen, Lina Gruzinskyte, Steffen Lynge Jørgensen, Anja Boisen, and Sarvesh Kumar Srivastava. An Ingestible Self-Polymerizing System for Targeted Sampling of Gut Microbiota and Biomarkers. ACS Nano, 14(9):12072-12081, sep 2020. ISSN 1936086X. doi: 10.1021/ACSNANO.0C05426/SUPPL_FILE/ NN0C05426_SI_001.MP4. URL https://pubs.acs.org/doi/full/10.1021/acsnano.0c05426.

Gastone Ciuti, Karolina Skonieczna-żydecka, Wojciech Marlicz, Veronica Iacovacci, Hongbin Liu, Danail Stoyanov, Alberto Arezzo, Marcello Chiurazzi, Ervin Toth, Henrik Thorlacius, Paolo Dario, and Anastasios Koulaouzidis. Frontiers of Robotic Colonoscopy: A Comprehensive Review of Robotic Colonoscopes and Technologies. Journal of Clinical Medicine 2020, Vol. 9, Page 1648, 9(6):1648, may 2020. ISSN 20770383. doi: 10.3390/JCM9061648. URL https://www.mdpi.com/2077-0383/9/6/1648/htmhttps: //www.mdpi.com/2077-0383/9/6/1648.

G. Cummins. Smart pills for gastrointestinal diagnostics and therapy. Advanced Drug Delivery Reviews, 177:113931, oct 2021. ISSN 0169-409X. doi: 10.1016/J.ADDR.2021.113931.

Gerard Cummins, Diana E. Yung, Ben F. Cox, Anastasios Koulaouzidis, Marc P.Y. Desmulliez, and Sandy Cochran. Luminally expressed gastrointestinal biomarkers. http://dx.doi.org/10.1080/17474124.2017.1373017, 11(12):1119-1134, dec 2017. ISSN 17474132. doi: 10.1080/17474124.2017.1373017. URL https://www.tandfonline.com/doi/abs/10.1080/17474124. 2017.1373017.

Raffaele Di Giacomo, Sebastian Krödel, Bruno Maresca, Patrizia Benzoni, Roberto Rusconi, Roman Stocker, and Chiara Daraio. Deployable micro-traps to sequester motile bacteria. Scientific Reports, 7(1):1-8, apr 2017. ISSN 20452322. doi: 10.1038/srep45897.

D. F. Evans, G. Pye, R. Bramley, A. G. Clark, T. J. Dyson, and J. D. Hardcastle. Measurement of gastrointestinal pH profiles in normal ambulant human subjects. Gut, 29(8):1035-1041, aug 1988. ISSN 0017-5749. doi: 10.1136/GUT.29.8.1035. URL https://gut.bmj . com/content/29/8/1035https://gut . bmj . com/content/29/8/1035. abstract.

David A. Giljohann and Chad A. Mirkin. Drivers of biodiagnostic development. Nature 2009 462:7272, 462 (7272):461-464, nov 2009. ISSN 1476-4687. doi: 10.1038/nature08605. URL https://www.nature.com/ articles/nature08605.

Deborah C. Hay Burgess, Jeffrey Wasserman, and Carol A. Dahl. Global health diagnostics. Nature 2006 444:1, 444(1):1-2, nov 2006. ISSN 1476-4687. doi: 10.1038/nature05440. URL https://www.nature. com/articles/nature05440.

Wenqi Hu, Guo Zhan Lum, Massimo Mastrangeli, and Metin Sitti. Small-scale soft-bodied robot with multimodal locomotion. Nature 2018 554:7690, 554(7690):81-85, jan 2018. ISSN 1476-4687. doi: 10.1038/ nature25443. URL https://www.nature.com/articles/nature25443.

Veronica Iacovacci, Izadyar Tamadon, Emanuele Federico Kauffmann, Stefano Pane, Virginia Simoni, Leonardo Marziale, Michele Aragona, Luigi Cobuccio, Massimo Chiarugi, Paolo Dario, Stefano Del Prato, Leonardo Ricotti, Fabio Vistoli, and Arianna Menciassi. A fully implantable device for intraperitoneal drug delivery refilled by ingestible capsules. Science Robotics, 6(57):3328, aug 2021. ISSN 24709476. 
doi: 10.1126/SCIROBOTICS.ABH3328/SUPPL_FILE/SCIROBOTICS.ABH3328_MDAR_CHECKLIST. DOCX. URL https://www.science.org/doi/abs/10.1126/scirobotics .abh3328.

Gavriel Iddan, Gavriel Meron, Arkady Glukhovsky, and Paul Swain. Wireless capsule endoscopy. Nature 2000 405:6785, 405(6785):417-417, may 2000. ISSN 1476-4687. doi: 10.1038/35013140. URL https: //www.nature.com/articles/35013140.

Gabor Kosa and Patrick Hunziker. Small-Scale Robots in Fluidic Media. Advanced Intelligent Systems, 1 (7):1900035, nov 2019. ISSN 2640-4567. doi: 10.1002/aisy.201900035.

Bryan Laulicht, Nicholas J. Gidmark, Anubhav Tripathi, and Edith Mathiowitz. Localization of magnetic pills. Proceedings of the National Academy of Sciences of the United States of America, 108(6):22522257, feb 2011. ISSN 00278424. doi: 10.1073/PNAS.1016367108/-/DCSUPPLEMENTAL. URL https: //www. pnas.org/content/108/6/2252https://www.pnas.org/content/108/6/2252. abstract.

Jihun Lee, Dong-in Kim, Seungmin Bang, and Sukho Park. Drug-Loaded Mucoadhesive Patch with Active Delivery and Controlled Releasing Ability. Advanced Intelligent Systems, page 2100203, jan 2022. ISSN 2640-4567. doi: 10.1002/AISY.202100203. URL https://onlinelibrary.wiley.com/doi/full/10. 1002/aisy. 202100203https://onlinelibrary.wiley.com/doi/abs/10.1002/aisy. 202100203https: //onlinelibrary.wiley.com/doi/10.1002/aisy. 202100203.

Dekey Y. Lhewa and Lisa L. Strate. Pros and cons of colonoscopy in management of acute lower gastrointestinal bleeding. World Journal of Gastroenterology, 18(11):1185-1190, 2012. ISSN 10079327. doi: 10.3748/wjg.v18.i11.1185. URL https://www.wjgnet.com/1007-9327/full/v18/i11/1185.htm.

Jinxing Li, Soracha Thamphiwatana, Wenjuan Liu, Berta Esteban-Fernández De Ávila, Pavimol Angsantikul, Elodie Sandraz, Jianxing Wang, Tailin Xu, Fernando Soto, Valentin Ramez, Xiaolei Wang, Weiwei Gao, Liangfang Zhang, and Joseph Wang. Enteric Micromotor Can Selectively Position and Spontaneously Propel in the Gastrointestinal Tract. ACS Nano, 10(10):9536-9542, oct 2016. ISSN 1936086X. doi: 10.1021/acsnano.6b04795.

Xinyue Liu, Christoph Steiger, Shaoting Lin, German Alberto Parada, Ji Liu, Hon Fai Chan, Hyunwoo Yuk, Nhi V. Phan, Joy Collins, Siddartha Tamang, Giovanni Traverso, and Xuanhe Zhao. Ingestible hydrogel device. Nature Communications 2019 10:1, 10(1):1-10, jan 2019. ISSN 2041-1723. doi: 10.1038/s41467019-08355-2. URL https://www.nature.com/articles/s41467-019-08355-2.

Xinyue Liu, Yueying Yang, Maria Eugenia Inda, Shaoting Lin, Jingjing Wu, Yoonho Kim, Xiaoyu Chen, Dacheng Ma, Timothy K Lu, Xuanhe Zhao, X Liu, Y Yang, S Lin, J Wu, Y Kim, X Chen, $\mathrm{X}$ Zhao, M E Inda, D Ma, and $\mathrm{T} \mathrm{K}$ Lu. Magnetic Living Hydrogels for Intestinal Localization, Retention, and Diagnosis. Advanced Functional Materials, 31(27):2010918, jul 2021. ISSN 16163028. doi: 10.1002/ADFM.202010918. URL https://onlinelibrary.wiley.com/doi/full/10. 1002/adfm. 202010918https://onlinelibrary.wiley.com/doi/abs/10.1002/adfm.202010918https: //onlinelibrary.wiley.com/doi/10.1002/adfm.202010918.

Nikolaj Kofoed Mandsberg, Juliane Fjelrad Christfort, Khorshid Kamguyan, Anja Boisen, and Sarvesh Kumar Srivastava. Orally ingestible medical devices for gut engineering. Advanced Drug Delivery Reviews, 165-166:142-154, jan 2020. ISSN 0169-409X. doi: 10.1016/J.ADDR.2020.05.004.

Mark Mimee, Phillip Nadeau, Alison Hayward, Sean Carim, Sarah Flanagan, Logan Jerger, Joy Collins, Shane McDonnell, Richard Swartwout, Robert J. Citorik, Vladimir Bulović, Robert Langer, Giovanni Traverso, Anantha P. Chandrakasan, and Timothy K. Lu. An ingestible bacterial-electronic system to monitor gastrointestinal health. Science, 360(6391):915-918, may 2018. ISSN 10959203. doi: 10.1126/ SCIENCE.AAS9315/SUPPL_FILE/AAS9315_TABLE_S3.XLSX. URL https ://www.science.org/doi/ abs/10.1126/science. aas9315.

Jihong Min, Yiran Yang, Zhiguang Wu, and Wei Gao. Robotics in the Gut. Advanced Therapeutics, page 
1900125, sep 2019. ISSN 2366-3987. doi: 10.1002/adtp.201900125. URL https://onlinelibrary.wiley . com/doi/abs/10.1002/adtp.201900125.

A. Nemiroski, M. Ryou, C. C. Thompson, and R. M. Westervelt. Swallowable fluorometric capsule for wireless triage of gastrointestinal bleeding. Lab on a Chip, 15(23):4479-4487, nov 2015. ISSN 14730189. doi: 10.1039/C5LC00770D. URL https://pubs.rsc.org/en/content/articlehtml/2015/lc/ c5lc00770dhttps://pubs.rsc.org/en/content/articlelanding/2015/lc/c5lc00770d.

Mehmet O. Ozen, Kaushik Sridhar, Mehmet Giray Ogut, Akash Shanmugam, Anirudh S. Avadhani, Yukari Kobayashi, Joseph C. Wu, Francois Haddad, and Utkan Demirci. Total Microfluidic chip for Multiplexed diagnostics (ToMMx). Biosensors and Bioelectronics, 150:111930, feb 2020. ISSN 18734235. doi: 10.1016/ j.bios.2019.111930.

Panpan Qiao, Hongying Liu, Xueping Yan, Ziru Jia, and Xitian Pi. A Smart Capsule System for Automated Detection of Intestinal Bleeding Using HSL Color Recognition. PLOS ONE, 11(11):e0166488, nov 2016. ISSN 1932-6203. doi: 10.1371/JOURNAL.PONE.0166488. URL https://journals.plos .org/plosone/ article?id=10.1371/journal. pone. 0166488 .

David F. Ransohoff. Rules of evidence for cancer molecular-marker discovery and validation. Nature Reviews Cancer 2004 4:4, 4(4):309-314, 2004. ISSN 1474-1768. doi: 10.1038/nrc1322. URL https://www .nature. com/articles/nrc1322.

Hojatollah Rezaei Nejad, Bruno C. M. Oliveira, Aydin Sadeqi, Amin Dehkharghani, Ivanela Kondova, Jan A. M. Langermans, Jeffrey S. Guasto, Saul Tzipori, Giovanni Widmer, and Sameer R. Sonkusale. Ingestible Osmotic Pill for In Vivo Sampling of Gut Microbiomes. Advanced Intelligent Systems, 1(5):1900053, sep 2019. ISSN 2640-4567. doi: 10.1002/aisy.201900053. URL https://onlinelibrary.wiley.com/doi/ abs/10.1002/aisy. 201900053.

Melany Rios-Morales, Mara P.H. van Trijp, Christiane Rösch, Ran An, Theo Boer, Albert Gerding, Naomi de Ruiter, Martijn Koehorst, M. Rebecca Heiner-Fokkema, Henk A. Schols, Dirk Jan Reijngoud, Guido J.E.J. Hooiveld, and Barbara M. Bakker. A toolbox for the comprehensive analysis of small volume human intestinal samples that can be used with gastrointestinal sampling capsules. Scientific Reports 2021 11:1, 11(1):1-14, apr 2021. ISSN 2045-2322. doi: 10.1038/s41598-021-86980-y. URL https://www . nature.com/articles/s41598-021-86980-y.

Aman Saini, Yash Pershad, Hassan Albadawi, Malia Kuo, Sadeer Alzubaidi, Sailendra Naidu, M-Grace Knuttinen, and Rahmi Oklu. Liquid Biopsy in Gastrointestinal Cancers. Diagnostics 2018, Vol. 8, Page 75, 8(4):75, oct 2018. ISSN 2075-4418. doi: 10.3390/DIAGNOSTICS8040075. URL https://www.mdpi. com/2075-4418/8/4/75/htmhttps://www.mdpi.com/2075-4418/8/4/75.

Mohammed E. Sayed, Jamie O. Roberts, Karen Donaldson, Stephen T. Mahon, Faiz Iqbal, Boyang Li, Santiago Franco Aixela, Georgios Mastorakis, Emil T. Jonasson, Markus P. Nemitz, Sara Bernardini, and Adam A. Stokes. Modular Robots for Enabling Operations in Unstructured Extreme Environments. Advanced Intelligent Systems, page 2000227, jan 2022. ISSN 2640-4567. doi: 10.1002/AISY.202000227. URL https://onlinelibrary.wiley.com/doi/full/10.1002/ aisy.202000227https://onlinelibrary.wiley.com/doi/abs/10.1002/aisy.202000227https:

//onlinelibrary.wiley.com/doi/10.1002/aisy. 202000227.

Vilayat Sayeed. Size, Shape, and Other Physical Attributes of Generic Tablets and Capsules Guidance for Industry. 2015. URL http://www.fda.gov/Drugs/GuidanceComplianceRegulatoryInformation/ Guidances/default.htm.

Sebastian Schostek, Melanie Zimmermann, Jan Keller, Mario Fode, Michael Melbert, Marc O. Schurr, Thomas Gottwald, and Ruediger L. Prosst. Telemetric real-time sensor for the detection of acute upper gastrointestinal bleeding. Biosensors and Bioelectronics, 78:524-529, apr 2016. ISSN 0956-5663. doi: 10.1016/J.BIOS.2015.11.073. 
Prima Dewi Sinawang, Fernando Soto, Mehmet Ozgun Ozen, Demir Akin, and Utkan Demirci. Progress and challenges in biomarker enrichment for cancer early detection. Progress in Biomedical Engineering, 3(4): 043001, sep 2021. doi: 10.1088/2516-1091/ac1ea3. URL https://doi.org/10.1088/2516-1091/ac1ea3.

Robert A. Smith, Vilma Cokkinides, Andrew C. von Eschenbach, Bernard Levin, Carmel Cohen, Carolyn D. Runowicz, Stephen Sener, Debbie Saslow, and Harmon J. Eyre. American Cancer Society Guidelines for the Early Detection of Cancer. CA: A Cancer Journal for Clinicians, 52(1):8-22, jan 2002. ISSN 15424863. doi: 10.3322/CANJCLIN.52.1.8. URL https://onlinelibrary.wiley.com/doi/full/10.3322/ canjclin.52.1.8https://onlinelibrary.wiley.com/doi/abs/10.3322/canjclin.52.1.8https:

//acsjournals.onlinelibrary.wiley.com/doi/10.3322/canjclin.52.1.8.

F. Soto, E. Karshalev, F. Zhang, B. Esteban Fernandez De Avila, A. Nourhani, and J. Wang. Smart Materials for Microrobots. Chemical Reviews, 2021. ISSN 15206890. doi: 10.1021/acs.chemrev.0c00999.

Fernando Soto, Jie Wang, Rajib Ahmed, and Utkan Demirci. Medical micro/nanorobots in precision medicine. Advanced Science, page 2002203, 2020. doi: 10.1002/advs.202002203.

Christoph Steiger, Alex Abramson, Phillip Nadeau, Anantha P. Chandrakasan, Robert Langer, and Giovanni Traverso. Ingestible electronics for diagnostics and therapy. Nature Reviews Materials 2018 4:2, 4(2):83-98, dec 2018. ISSN 2058-8437. doi: 10.1038/s41578-018-0070-3. URL https://www. nature.com/articles/ s41578-018-0070-3.

Qiang Tang, Ge Jin, Gang Wang, Tianyu Liu, Xiang Liu, Bangmao Wang, and Hailong Cao. Current Sampling Methods for Gut Microbiota: A Call for More Precise Devices. Frontiers in Cellular and Infection Microbiology, 10:151, apr 2020. ISSN 22352988. doi: 10.3389/FCIMB.2020.00151/BIBTEX.

Jiaobing Tu and Wei Gao. Spray-on magnetic skin for robotic actuation. Science Robotics, 5(48):1390, nov 2020. ISSN 24709476. doi: 10.1126/SCIROBOTICS.ABF1390/ASSET/B3B82B38-ECAC-4BA781E6-7E827870F156/ASSETS/GRAPHIC/ABF1390-F1.JPEG. URL https://www.science.org/doi/ abs/10.1126/scirobotics. abf 1390 .

Jose Fernando Waimin, Sina Nejati, Hongjie Jiang, Jake Qiu, Jianghsan Wang, Mohit S. Verma, and Rahim Rahimi. Smart capsule for non-invasive sampling and studying of the gastrointestinal microbiome. RSC Advances, 10(28):16313-16322, apr 2020. ISSN 2046-2069. doi: 10.1039/C9RA10986B. URL https://pubs.rsc.org/en/content/articlehtml/2020/ra/c9ra10986bhttps://pubs.rsc.org/ en/content/articlelanding/2020/ra/c9ra10986b.

R. J. Washabau, M. J. Day, M. D. Willard, E. J. Hall, A. E. Jergens, J. Mansell, T. Minami, and T. W. Bilzer. Endoscopic, Biopsy, and Histopathologic Guidelines for the Evaluation of Gastrointestinal Inflammation in Companion Animals. Journal of Veterinary Internal Medicine, 24(1):10-26, jan 2010. ISSN 1939-1676. doi: 10.1111/J.1939-1676.2009.0443.X. URL https://onlinelibrary.wiley.com/doi/full/ 10.1111/j.1939-1676.2009.0443.xhttps://onlinelibrary.wiley.com/doi/abs/10.1111/j.19391676.2009.0443.xhttps://onlinelibrary.wiley.com/doi/10.1111/j.1939-1676.2009.0443.x.

Chenxi Wu, Jiayin Zhang, Haibo Li, Wenrong $\mathrm{Xu}$, and $\mathrm{Xu}$ Zhang. The potential of liquid biopsies in gastrointestinal cancer. Clinical Biochemistry, 84:1-12, oct 2020. ISSN 0009-9120. doi: 10.1016/J. CLINBIOCHEM.2020.06.007.

Zhiguang Wu, Lei Li, Yiran Yang, Peng Hu, Yang Li, So Yoon Yang, Lihong V. Wang, and Wei Gao. A microrobotic system guided by photoacoustic computed tomography for targeted navigation in intestines in vivo. Science Robotics, 4(32), jul 2019. ISSN 24709476. doi: 10.1126/scirobotics.aax0613.

Ya Xiong, Lin Qi, Ye Niu, Yueqiang Lin, Qingzhong Xue, and Yi Zhao. Autonomous Drug Release Systems with Disease Symptom-Associated Triggers. Advanced Intelligent Systems, 2(3):1900124, mar 2020. ISSN 2640-4567. doi: 10.1002/AISY.201900124. URL https://onlinelibrary.wiley.com/doi/full/10. 1002/aisy. 201900124https://onlinelibrary.wiley.com/doi/abs/10.1002/aisy.201900124https: //onlinelibrary.wiley.com/doi/10.1002/aisy.201900124. 
Changyu Xu, Zilin Yang, Shaun Wee, Kiat Tan, Jianhuang Li, and Guo Zhan Lum. Magnetic Miniature Actuators with Six-Degrees-of-Freedom Multimodal Soft-Bodied Locomotion. Advanced Intelligent Systems, page 2100259, jan 2022. ISSN 2640-4567. doi: 10.1002/AISY.202100259. URL https://onlinelibrary. wiley.com/doi/full/10.1002/aisy.202100259https://onlinelibrary.wiley.com/doi/abs/10. 1002/aisy. 202100259https://onlinelibrary.wiley.com/doi/10.1002/aisy. 202100259.

Sehyuk Yim, Evin Gultepe, David H. Gracias, and Metin Sitti. Biopsy using a magnetic capsule endoscope carrying, releasing, and retrieving untethered microgrippers. IEEE Transactions on Biomedical Engineering, 61(2):513-521, feb 2014. ISSN 00189294. doi: 10.1109/TBME.2013.2283369.

Ke Yuan, Elya A. Shamskhou, Mark E. Orcholski, Abinaya Nathan, Sushma Reddy, Hiroaki Honda, Vigneshwaran Mani, Yitian Zeng, Mehmet O. Ozen, Lingli Wang, Utkan Demirci, Wen Tian, Mark R. Nicolls, and Vinicio A. De Jesus Perez. Loss of Endothelium-Derived Wnt5a Is Associated with Reduced Pericyte Recruitment and Small Vessel Loss in Pulmonary Arterial Hypertension. Circulation, 139(14):1710-1724, apr 2019. ISSN 15244539. doi: 10.1161/CIRCULATIONAHA.118.037642. URL https://www . ahajournals.org/doi/abs/10.1161/CIRCULATIONAHA.118.037642. 